\title{
Kein Vorteil für die lokale Kontrolle nach Dosiseskalation der Strahlentherapie bei Patienten mit Ösophaguskarzinom nach einer definitiven Radiochemotherapie: randomisierte Phase-III-Studie ARTDECO
}

\author{
Emmanouil Fokas ${ }^{1}$ - Daniel Martin ${ }^{2}$. Claus Rödel ${ }^{2}$ \\ Angenommen: 3. Februar 2022 / Online publiziert: 15. Februar 2022 \\ (c) Der/die Autor(en) 2022
}

Hintergrund In der randomisierten, klinischen Phase-IIIStudie ARTDECO wurde bei Patient*innen mit lokal fortgeschrittenem Ösophaguskarzinom die Rolle der Radiotherapiedosiseskalation auf den Primärtumor im Hinblick auf die lokale Tumorkontrolle nach definitiver Radiochemotherapie $(\mathrm{RCT})$ untersucht.

Methode Patient*innen mit funktionell inoperablem und/ oder irresektablem Ösophaguskarzinom wurden eingeschlossen. Die Randomisierung erfolgte in einen Arm mit einer Gesamtdosis von 50,4 Gy/1,8 Gy auf den Primärtumor oder alternativ einer Dosiseskalation bis zu einer Gesamtdosis von $61,6 \mathrm{~Gy} / 2,2 \mathrm{~Gy}$. Die regionären Lymphknoten wurden in beiden Gruppen mit 50,4 Gy/1,8 Gy bestrahlt. Die simultane Chemotherapie bestand in beiden Armen aus Carboplatin (AUC2) und Paclitaxel $\left(50 \mathrm{mg} / \mathrm{m}^{2}\right)$ einmal wöchentlich über 6 Wochen. Der primäre Endpunkt war das lokale progressionsfreie Überleben (LPFS).

Ergebnisse Von September 2012 bis Juni 2018 wurden 260 Patient*innen eingeschlossen; $61 \%$ hatten ein Plattenepithelkarzinom, 39\% ein Adenokarzinom. Eine vollständige Strahlenbehandlung (RT) erhielten insgesamt $94 \%$ der Patient*innen, bei $85 \%$ konnten mindestens 5 Chemothera-

\section{Originalpublikation Hulshof M, Geijsen ED, Rozema T et al (2021) Randomized Study on Dose Escalation in Definitive Chemoradiation for Patients With Locally Advanced Esophageal Cancer (ARTDECO Study). J Clin Oncol 39(25):2816-2824. https://doi.org/10.1200/JCO.20.03697.}

Prof. Dr. med. Dr. Emmanouil Fokas emmanouil.fokas@kgu.de

1 Klinik für Strahlentherapie und Onkologie, Universitätsklinikum Frankfurt, Goethe-Universität Frankfurt, Theodor-Stern-Kai 7, 60590 Frankfurt/M., Deutschland

2 Frankfurt/M., Deutschland piezyklen verabreicht werden. Nach einer medianen Nachbeobachtungszeit von 50 Monaten betrug das lokale progressionsfreie Überleben nach 3 Jahren $70 \%$ in der Standarddosisgruppe gegenüber $73 \%$ in der Dosiseskalationsgruppe (Unterschied nicht signifikant). Das LPFS erreichte bei Plattenepithelkarzinomen $75 \%$ bzw. $79 \%$, und bei Adenokarzinomen $61 \%$ bzw. $61 \%$ nach 3 Jahren (Unterschiede nicht signifikant). Das lokoregionäre progressionsfreie Überleben betrug nach 3 Jahren $52 \%$ im Arm mit der Standarddosis und 59\% in der Dosiseskalationsgruppe $(p=0,08)$. Die Grad-4- und -5-Akuttoxizität erreichte $12 \%$ bzw. $5 \%$ im Standarddosisarm gegenüber $14 \%$ bzw. $10 \%$ im Dosiseskalationsarm $(p=0,15)$.

Schlussfolgerung der Autoren Bei Patient*innen mit fortgeschrittenem Ösophaguskarzinom, die mit einer definitiven Radiochemotherapie behandelt wurden, führte eine Dosiseskalation auf 61,6 Gy zu keiner signifikanten Verbesserung der lokalen Kontrolle im Vergleich zu 50,4Gy. Der fehlende Nutzen der Dosiseskalation wurde für beide Histologien beobachtet.

\section{Kommentar}

Verschiedene Dosierungsschemata wurden für die definitive RCT von Ösophaguskarzinomen untersucht [1]. Historisch zeigte die RTOG-85-01-Studie signifikant verbesserte Gesamtüberlebensraten nach RCT mit 50 Gy/2 Gy im Vergleich zur alleinigen Radiotherapie (RT) mit 64Gy/2 Gy [2]. Die lokoregionäre Rezidivrate/Progressionsrate betrug hierbei allerdings $47 \%$. Die hohe Rate an Lokalrezidiven/ Progressionen nach definitiver RCT bis 50 Gy ließ vermuten, dass höhere Bestrahlungsdosen die lokale Kontrolle potenziell weiter verbessern würden. Dies führte zur INT0123-Phase-III-Studie, bei der Patient*innen eine RCT ent- 
weder mit 50,4 Gy/1,8 Gy oder mit 64,8 Gy/1,8 Gy erhielten [3]. Auch in dieser Studie zeigte sich kein Unterschied zur lokalen/lokoregionären Tumorkontrolle oder dem Überleben in beiden Armen. Obwohl in der Hochdosisgruppe 11 behandlungsbedingte Todesfälle auftraten, verglichen mit zwei in der Standarddosisgruppe, traten 7 der 11 Todesfälle bei Patient*innen auf, die 50,4 Gy oder weniger erhalten hatten. Relativierend darf aber bemerkt werden, dass die INT-0123-Studie vor der Einführung moderner Strahlentherapietechniken durchgeführt wurde, was die hohen Letalitätsraten zumindest zum Teil erklärt.

In der hier kommentierten, randomisierten Phase-III-Studie ARTDECO wurde nach einer medianen Nachbeobachtungszeit von 50 Monaten der primäre Endpunkt des lokal progressionsfreien Überlebens (LPFS) nach einer Dosiseskalation der Strahlentherapie von 50,4 Gy auf 61,6 Gy auf den Primärtumor nicht signifikant verbessert [4]. Einige Aspekte der Studie sind deshalb hier erwähnenswert. In der ARTDECO-Studie waren die Staging- und Strahlentherapietechniken auf dem neuesten Stand. Das Fehlen eines Vorteils in Bezug auf die lokale Kontrolle nach einer Dosiseskalation über 50,4 Gy hinaus ist daher bemerkenswert. Obwohl in der Dosiseskalationsgruppe ein leichter Anstieg der toxizitätsbedingten Todesfälle beobachtet wurde, erklärt dies nicht die fehlende Verbesserung der lokalen Tumorkontrolle. Die niedriger dosierten Bestrahlungsvolumina deckten die regionalen Lymphknoten im Vergleich zu dem in der CROSS-Studie verwendeten Bestrahlungsprotokoll adäquat $a b$.

Anders als beim nichtkleinzelligen Lungenkarzinom [5] wird bei Patient*innen mit Ösophaguskarzinom nach wie vor eine Bestrahlung der elektiven Lymphknoten empfohlen, auch wenn im Rahmen des Stagings eine FDG-PET/CT durchgeführt wurde. Es gibt aber zunehmend Hinweise darauf, dass die elektive Bestrahlung von Lymphknoten die Immunantwort gegen den Tumor beeinträchtigen könnte [6-8], obwohl zur Erhärtung dieser Vermutung weitere Studien erforderlich sind. Darüber hinaus wurde die Studie kritisiert, weil ihre statistische Teststärke („Power“) für den primären Endpunkt das notwendige Maß unterschritt (,underpowered"), was die Zuverlässigkeit der Ergebnisse beeinträchtigen könnte [9].

\section{Fazit}

Die Verbesserung der lokalen Kontrolle ist nach wie vor ein wichtiges Ziel bei der Behandlung von Ösophaguskarzinomen. Die Evidenz aus den Studienergebnissen von RTOG 85-01, INT 0123 und nun auch ARTDECO spricht beim fortgeschrittenen Ösophaguskarzinom für eine erforderliche Gesamtdosis von 50,4Gy in 28 Fraktionen als Standarddosis, wenn die Patient*innen mit einer definitiven
RCT behandelt werden. Die Ergebnisse der noch laufenden Dosiseskalationsstudien (ClinicalTrials.gov-Identifikator: NCT01348217, NCT02741856 und NCT02556762) stehen noch aus und werden weitere randomisierte Daten zur Frage der RT-Dosiseskalation beim Ösophaguskarzinom liefern.

\section{Emmanouil Fokas, Daniel Martin, Claus Rödel, Frankfurt/M.}

Funding Open Access funding enabled and organized by Projekt DEAL.

Interessenkonflikt E. Fokas, D. Martin und C. Rödel geben an, dass kein Interessenkonflikt besteht.

Open Access Dieser Artikel wird unter der Creative Commons Namensnennung 4.0 International Lizenz veröffentlicht, welche die Nutzung, Vervielfältigung, Bearbeitung, Verbreitung und Wiedergabe in jeglichem Medium und Format erlaubt, sofern Sie den/die ursprünglichen Autor(en) und die Quelle ordnungsgemäß nennen, einen Link zur Creative Commons Lizenz beifügen und angeben, ob Änderungen vorgenommen wurden.

Die in diesem Artikel enthaltenen Bilder und sonstiges Drittmaterial unterliegen ebenfalls der genannten Creative Commons Lizenz, sofern sich aus der Abbildungslegende nichts anderes ergibt. Sofern das betreffende Material nicht unter der genannten Creative Commons Lizenz steht und die betreffende Handlung nicht nach gesetzlichen Vorschriften erlaubt ist, ist für die oben aufgeführten Weiterverwendungen des Materials die Einwilligung des jeweiligen Rechteinhabers einzuholen.

Weitere Details zur Lizenz entnehmen Sie bitte der Lizenzinformation auf http://creativecommons.org/licenses/by/4.0/deed.de.

\section{Literatur}

1. Shah MA, Kennedy EB, Catenacci DV, Deighton DC, Goodman KA, Malhotra NK, Willett C, Stiles B, Sharma P, Tang L, Wijnhoven BPL, Hofstetter WL (2020) Treatment of Locally Advanced Esophageal Carcinoma: ASCO Guideline. J Clin Oncol 38(23):2677-2694. https://doi.org/10.1200/JCO.20.00866

2. Cooper JS, Guo MD, Herskovic A, Macdonald JS, Martenson JA Jr., Al-Sarraf M, Byhardt R, Russell AH, Beitler JJ, Spencer S, Asbell SO, Graham MV, Leichman LL (1999) Chemoradiotherapy of locally advanced esophageal cancer: long-term follow-up of a prospective randomized trial (RTOG 85-01). Radiation Therapy Oncology Group. JAMA 281(17):1623-1627. https://doi.org/10.1001/ jama.281.17.1623

3. Minsky BD, Pajak TF, Ginsberg RJ, Pisansky TM, Martenson J, Komaki R, Okawara G, Rosenthal SA, Kelsen DP (2002) INT 0123 (Radiation Therapy Oncology Group 94-05) phase III trial of combined-modality therapy for esophageal cancer: high-dose versus standard-dose radiation therapy. J Clin Oncol 20(5):1167-1174. https://doi.org/10.1200/JCO.2002.20.5.1167

4. Hulshof M, Geijsen ED, Rozema T, Oppedijk V, Buijsen J, Neelis KJ, Nuyttens J, van der Sangen MJC, Jeene PM, Reinders JG, van Berge Henegouwen MI, Thano A, van Hooft JE, van Laarhoven HWM, van der Gaast A (2021) Randomized study on dose escalation in definitive chemoradiation for patients with locally advanced esophageal cancer (ARTDECO study). J Clin Oncol 39(25):2816-2824. https://doi.org/10.1200/JCO.20.03697

5. Nestle U, Schimek-Jasch T, Kremp S, Schaefer-Schuler A, Mix M, Kusters A, Tosch M, Hehr T, Eschmann SM, Bultel YP, Hass P, Fleckenstein J, Thieme A, Stockinger M, Dieckmann K, Miede- 
rer M, Holl G, Rischke HC, Gkika E, Adebahr S, Konig J, Grosu AL, group PE-Ps (2020) Imaging-based target volume reduction in chemoradiotherapy for locally advanced non-small-cell lung cancer (PET-Plan): a multicentre, open-label, randomised, controlled trial. Lancet Oncol 21(4):581-592. https://doi.org/10.1016/S14702045(20)30013-9

6. Marciscano AE, Ghasemzadeh A, Nirschl TR, Theodros D, Kochel CM, Francica BJ, Muroyama Y, Anders RA, Sharabi AB, Velarde E, Mao W, Chaudhary KR, Chaimowitz MG, Wong J, Selby MJ, Thudium KB, Korman AJ, Ulmert D, Thorek DLJ, DeWeese TL, Drake CG (2018) Elective nodal irradiation attenuates the combinatorial efficacy of stereotactic radiation therapy and immunotherapy. Clin Cancer Res 24(20):5058-5071. https://doi.org/10. 1158/1078-0432.CCR-17-3427
7. Buchwald ZS, Nasti TH, Lee J, Eberhardt CS, Wieland A, Im SJ, Lawson D, Curran W, Ahmed R, Khan MK (2020) Tumor-draining lymph node is important for a robust abscopal effect stimulated by radiotherapy. J Immunother Cancer. https://doi.org/10.1136/ jitc-2020-000867

8. Qian JM, Schoenfeld JD (2020) Radiotherapy and immunotherapy for head and neck cancer: current evidence and challenges. Front Oncol 10:608772. https://doi.org/10.3389/fonc.2020.608772

9. Pottgen C, Nestle U, Hocht S, Stuschke M (2021) Interactions between dose and volume in chemoradiotherapy of esophageal cancer. J Clin Oncol 39(34):3880-3881. https://doi.org/10.1200/JCO. 21.01579 